\title{
LA DISCULPA CORPORATIVA A TRAVÉS DE TWITTER EN IBERIA Y BANCO SANTANDER
}

\section{THE CORPORATE APOLOGY VIA TWITTER FROM IBERIA AND BANCO SANTANDER}

\author{
Ana M. Fernández-VAllejo \\ ISSA-School of Managment Assistant \\ Instituto Cultura y Sociedad (ICS) \\ Universidad de Navarra \\ Email: amf@unav.es
}

Recibido: 01/09/2017

Aceptado: 02/11/2017

\section{Resumen}

En los últimos años las compañías han creado nuevos canales de atención al cliente sirviéndose de las distintas redes sociales, entre ellas Twitter. El objetivo de este trabajo es analizar cómo las compañías expresan sus disculpas a través de Twitter. Se propone un análisis lingüísticodiscursivo de la disculpa corporativa, apoyados en la lingüística de corpus (Page, 2014) y la pragmática de la disculpa (Harris et alii, 2006). El análisis se aplicará a un corpus conformado por los 1567 tuits publicados durante la primera quincena de julio de 2017 por Iberia y Banco Santander; dos compañías que cotizan en el Ibex 35 y destacan por la atención al cliente ofrecida a través de este canal. Las dos compañías coinciden en una estructura común de tuits, así como en el reconocimiento directo o indirecto de la ofensa cometida; sin embargo, el Banco Santander frente a la compañía Iberia presenta un estilo más burocrático y alejado, omitiendo explicaciones.

PALABRAS CLAVE: Disculpa; Discurso corporativo; Twitter; Banco Santander; Iberia; Servicio al cliente.

\begin{abstract}
In recent years companies have created new customer channels making use of the various social networks, including Twitter. The aim of this paper is to analyze how companies express their apology via Twitter. This paper offers a linguistic-discursive analysis of corporate apologies, supported by corpus linguistics (Page, 2014) and a further pragmatic analysis of the apology (Harris et alii, 2006). The analysis is applied to a body made up of 1567 tweets published during the first half of July 2017 by Iberia and Banco Santander; two companies listed on the Ibex 35 which stand out for their attention to customers offered through this channel. The two companies utilize a common structure of tweets, as well as direct or indirect recognition of the offense; however, Banco Santander has a more bureaucratic and distant style than Iberia, whereby omitting the explanations.

KEYWORDS: Apologies; Corporate Discourse; Twitter; Banco Santander; Iberia; Customer Service.
\end{abstract}

Para citar este artículo / To cite this article: Fernández-Vallejo, Ana M. (2017). La disculpa corporativa a través de Twitter en Iberia y Banco Santander. ELUA, 31: 151-170. doi: 10.14198/ELUA2017.31.08

Enlace / Link: http://dx.doi.org/10.14198/ELUA2017.31.08 


\section{INTRODUCCIÓN}

La atención al cliente ha venido generando en muchas ocasiones emociones negativas y recelos. Aunque, en origen, se ideó para ayudar al consumidor, la espera en el teléfono o la llegada de un correo impersonal o una mala cara o una palabra inoportuna generaba en muchos casos más daños que beneficios a la relación entre compañías y cliente (Couso, 2005). Sin embargo, los medios sociales y, en concreto, Twitter, y después Facebook han resuelto, en parte, estos problemas. Ahora se ofrece un servicio que no solo mejora el existente sino que además reduce costes: la respuesta es más rápida y frecuente; se disminuye el número de personal en atención al cliente y el servicio ofrecido se aumenta, en muchas ocasiones, hasta alcanzar los siete días de las semana durante 24 horas (Parr, 2009; Culnan et alii, 2010; Flynn, 2016).

Un aspecto importante de este nuevo canal de atención al cliente es su carácter público. Las nuevas tecnologías sociales permiten que los mensajes de clientes enfadados lleguen a ser leídos por miles de lectores, pero también las respuestas posteadas a través de estos medios permite a las compañías comunicar más allá del cliente particularmente afectado. El rasgo exclusivamente dialógico (cliente-empresa) de los medios tradicionales, como el correo o el teléfono, desaparecen (Thornborrow y Mongtgomery, 2010). De hecho, el impacto de estos mensajes, tanto los enviados por el cliente como los publicados por la empresa, son mucho más transcendentes de lo que podemos imaginar y pueden ser evaluados por un público muy amplio.

Las respuestas a los posts y tuits de los clientes -cargados habitualmente de preocupación, en unos casos; otros, enfado- no solamente son un mero intercambio de mensajes para solucionar un problema puntual, en juego está la identidad corporativa y la reputación de marca. Las relaciones de confianza y lealtad pueden verse erosionadas hasta romperse el engagement entre cliente y compañía (Creelman, 2014). Está ya demostrado que una experiencia positiva con la marca a través de las redes sociales lleva a una mayor fidelidad y este deriva, finalmente, en un mayor número de compras (Hudson et alii, 2015), de ahí que cada vez más las empresas incluyan dentro de su estrategia de comunicación digital este canal de atención al cliente, desde donde el clienten puede obtener de un modo sencillo y rápido una retroalimentación de la compañía (Schultz et alii, 2011).

Esta realidad sugiere que el escenario público de Twitter ofrece un poder inimaginable al cliente (Hutchby, 2001). La posibilidad de hacer pública una queja -por pequeña que seapuede provocar un daño terrible en la imagen de la empresa con consecuencias imprevisibles (Benoit, 2014). Se puede recordar a este respecto ejemplos, en los que una respuesta tardía a la publicación de una queja trae daños irreparable ya no solo para la imagen de la compañía, sino también para su cotización en los mercados financieros (Cosh, 2009). Estamos ante crisis de comunicación, que no han obtenido las respuestas adecuadas en el momento oportuno. La investigación de las crisis de comunicación tanto en medios sociales como en otro tipo de canal (televisión o periódicos) ha recibido una gran atención por parte de la investigación (Coombs, 2007; Schultz, 2011).

En este artículo nos centraremos en cómo las empresas expresan la disculpa en el contexto digital que ofrece Twitter. Este microblog aporta un espacio excepcional a compañías y clientes. La persona que se siente vulnerada por algún motivo expresa su queja públicamente a través de Twitter; para que la queja llegue a la persona física o jurídica que causó el perjuicio será necesario que en el post sea mencionado; es decir, se ha de incluir su nombre de usuario 
en Twitter precedido de la arroba@mención, que viene a comportarse como un vocativo (Zappavigna, 2011). De esta manera la queja queda reflejada por partida doble: en el timeline de la persona que realiza la queja de Twitter y, al mismo tiempo, en el de la empresa. Una vez que la empresa recibe la notificación, puede contestar gracias a la función responder; se genera, entonces, un tuit que incluye automáticamente los nombres de los usuarios implicados y se publica también en ambos perfiles. La propia arquitectura de Twitter permite seguir la queja y disculpa no solo a los perfiles implicados, sino a cualquier seguidor de cualquiera de las dos cuentas, siempre y cuando acceda a sus perfiles directamente. Veamos a continuación un ejemplo, donde el cliente expresa su enfado por el trato recibido:

(1)@Iberia Que mal servicio del call center @Iberia, siempre hago el check in por el portal y ahora no puedo

Una hora después, la compañía responde con un mensaje conciliador:

(2)@username Hola Roberto, desconocemos qué ha podido haber pasado, estamos a tu disposición por DM. Saludos

Esta investigación propone un análisis pragmático de la noción de disculpa en la atención al cliente ofrecida por dos cuentas corporativas de Twitter. El análisis así planteado desea responder a las siguientes preguntas de investigación:

1. ¿Cuáles son las estrategias comunicativas de disculpas utilizadas en cada una de las cuentas de Twitter analizadas?

2. ¿Cuáles son las fórmulas habituales en dichas cuentas?

3. ¿El comportamiento de la cuentas corporativas de Twitter respecto a las disculpas es el mismo en compañías de diferentes sectores?

En este sentido, esperamos que nuestra investigación centrada en la aproximación lingüístico-discursiva, ahonde en la expresión de la cortesía lingüística en la empresa y, al mismo tiempo, complete otros estudios sobre la disculpa corporativa enmarcados en otras áreas, como las relaciones públicas, comunicación digital corporativa y la comunicación de crisis en las empresas.

Para responder a estar preguntas, el contenido de este artículo se organiza en tres partes: en la primera, se presenta el marco teórico, corpus y metodología que se va a utilizar para realizar el estudio previsto; en la segunda, se discuten los resultados obtenidos y en la tercera, se sintetizan las conclusiones e implicaciones pedagógicas que se desprende del presente trabajo.

\section{PRESENTE ESTUDIO. MARCO TEÓRICO, CORPUS Y METODOLOGÍA}

\subsection{Marco teórico. Aproximación discursivo-lingüística a las disculpas}

La disculpa como acto de habla ha generado una abundante bibliografía desde que Meier publicara en 1998 el artículo clave Apologies: What Do We Know?; dichos estudios se han interesado por la disculpa a través de distintas enfoques y disciplinas (pragmática, 
sociolingüística, psicología social, filosofía o enseñanza de lenguas extranjeras) ${ }^{1}$. Encontramos estudios que se centran en las características de la disculpa como acto de habla (Blum Kulka et alii, 1989; Tanaka et alii, 2008; Shariati and Chamani, 2010); otros se han fijado en el estilo comunicativo usado para las disculpas, bien sea directa o indirecta (Mills, 2003; Rundquist, 2007; Jucker and Taavitsainen, 2008). Otros lo abordan como estrategias de influencias, subrayando los riesgos de reputación bien para la persona que presenta la disculpa (Ogiermann, 2009) bien para las relaciones comerciales (Pace et alii, 2010). En este sentido, se han realizado estudios sobre todo en el ámbito privado y, en menor medida, en el público (Harris et alii, 2006; Kampf, 2009; Gruber, 2011).

Sin embargo, el casi novedoso uso de Twitter como canal de disculpas explica la escasa atención que ha recibido la disculpa corporativa en el ámbito sociolingüístico y pragmático a través de esta red (Park et alii, 2011; Page et alii, 2014). La investigación sobre disculpa que toma como foco de atención las redes sociales, al margen de las crisis de comunicación, ha dedicado sus estudios más bien a perfiles de figuras destacadas de los ámbitos: famosos del mundo del deporte (Hull y Lewis 2014), políticos (Ott, 2017) y gurús del mundo empresarial (Kim et alii, 2015), pero estos últimos estudios no se han centrado en cuestiones lingüísticas sino fundamentalmente en el éxito o no de la disculpa.

Respecto a qué es una disculpa y su clasificación (Harris et alii, 2006), casi podemos encontrar una definición y una taxonomía distinta por cada una de las disciplinas y su diferente objeto de estudio. Pero conviene aportar, al menos, una definición de trabajo, que en nuestro caso se apoyará en la pragmática lingüística y en la teoría de la cortesía, a la que la disculpa ha estado tradicionalmente ligada (Deutschmann, 2003; Unceta, 2014).

En suma, este estudio explorará la pragmática de la apología corporativa como un tipo de discurso con unas características concretas, analizando una muestra de ejemplos generada en las cuentas de Twitter creadas por las compañías para la atención al cliente. Nos limitaremos, por tanto, a las disculpas corporativas que se generan entre compañía y cliente en el espacio público de Twitter, sin atender otro tipo de discurso que puede generarse también en este tipo de cuentas.

Adaptando los estudios previos sobre disculpa (Holmes, 1998: 204), definimos la disculpa corporativa como un acto lingüístico en el que se intenta subsanar una posible ofensa cometida contra el cliente, para así regresar a una situación de entendimiento entre cliente y compañía; el primer objetivo de la disculpa es reestablecer la armonía perdida. Estamos ante un acto de habla ligado a la expresión de cortesía y, por tanto, enmarcado en un acto social, cultural y, en este caso, también corporativo.

Antes de abordar la metodología y dado el marco teórico generado sobre la disculpa en el ámbito corporativo y otros (Benoit, 2014), resulta útil identificar las características particulares de las disculpas corporativas.

1. Las disculpas corporativas se generan tras un conflicto o controversia entre compañía y empresa; son las denominadas corrective facework (Mok y Takunaga, 2009) y en raras ocasiones se genera a partir de situaciones en las que el cliente no ha llegado a expresarse. En la mayoría de los casos se trata de respuestas a demandas por parte del cliente que se siente víctima de una ofensa o una práctica injusta. La disculpa se entiende como intentos convencionales para mitigar el enfado del destinatario; en este caso, el cliente (Salgado,

1 El estudio de Ogierman (2009) revisa buena parte de la bibliografía publicada hasta esa fecha. 
2012: 215). Esta función reparadora es lo que distingue el acto de pedir disculpas de otros actos sociables como el de expresar agradecimiento o felicitaciones, donde la comunicación no requiere de una respuesta por parte del interlocutor.

2. A través de la disculpa corporativa, la empresa admite que ha cometido un error que afecta negativamente al cliente, pero también a la imagen de la empresa, erosionando su reputación de marca (Benoit, 2014). Por tanto, la disculpa es un acto de habla expresivo cuyo objeto ilocutivo es dar a conocer al cliente que se ha violado cierto contrato tácito y que la empresa se cree, al menos parcialmente, responsable de haber ocasionado dicha violación. Como señala Mills (2003: 62-70), estamos ante casos de negociación más o menos complejas entre las partes implicadas en la transacción comercial.

3. La disculpa corporativa está ligada a las estrategias de cortesía, dentro de la cual juega un papel preponderante el concepto de imagen de la empresa. La cortesía ha de ser vista no solo como un conjunto de fórmulas (Brown and Levinson, 1987) y estrategias comunicativas que se producen cuando los intereses de ambas partes no son coincidentes, sino como un acto de lengua que implica el concepto de lucha discursiva, que aúna al mismo tiempo argumentación y evaluación (Eelen, 2014: 240). Interesa, por tanto, ver la disculpa corporativa como un discurso argumentativo que trata de convencer al cliente de la posición tomada por la compañía, salvar su imagen y, en última instancia, mantener su confianza. En definitiva, es una acto social (Hargie et alii, 2010), pero también comercial.

4. Desde el punto de vista lingüístico, la disculpa corporativa, como el resto de diculpas, se sirve de diferentes estrategias comunicativas y formas lingüísticas. Entre las formas lingüísticas, cabe destacar los mecanismos de indicadores de la fuerza ilocutiva (a partir de ahora, MIFI), que presentan la disculpa de forma explícita como son los verbos performativos disculparse, perdonar, perdón (Tzu Yu, 2015); o fórmulas del tipo lo siento, pido disculpas, procedimientos léxicos que están altamente convencionalizados (Searle 1969: 64; Siebold, 2008: 50) y, de acuerdo con Harris et alii (2006), son de carácter obligatorio. A partir de esta premisa, enunciamos la hipótesis 1:

H1. Los MIFI presentarán una frecuencia elevada en los tuits de las dos compañías.

En el presente estudio queríamos ver hasta qué punto en función de las distintas compañías y sectores existía preferencias culturales corporativas que hacían que se optara por unas fórmulas u otras, y en qué grado se manifestaban.

Junto a estos mecanismos podemos encontrar otros recursos de carácter indirecto y opcionales como verbos modales, partículas e intensificadores tales como de verdad lo siento, cuánto lo siento, lo siento mucho o adverbios. Sin embargo, Mok y Tokunaga (2009), al tratar el modelo de la disculpa como acto de habla destacan que los intensificadores MIFI son más comunes en las conversaciones privadas que en los actos públicos. De este modo, enunciamos la hipótesis 2:

H2. En los tuits de las compañías el número de intensificadores será bajo.

En lo referente a las estrategias implícitas, podemos simplificar las distintas taxonomías existentes (Harris et alii, 2006), distinguiendo entre aquellos enunciados que hacen referencia las circunstancias previas a la disculpa y aquellos que hacen referencia a las 
circunstancias que siguen a la disculpa (Unceta, 2014). Dentro del primer grupo, estarían a) declaración de responsabilidad, implicando el pesar del causante y b) repetición del problema o error cometido, es lo que Holmes (1998) denomina, espacio para la ofensa; dentro del segundo, encontraríamos los mecanismos para restaurar el equilibrio y la relación entre los interlocutores: c) promesa de mejora, d) explicación de las causas, e) ofrecimiento de reparación, e) expresiones de autorreproche Fue por mi culpa; Yo soy el culpable, f) expresiones de remordimiento Me siento muy triste por lo que hice. De entre estos mecanismos, la aceptación de responsabilidad es obligatoria, según Harris et alii 2006. Así llegamos a la hipótesis 3.

H3. En todas las disculpas habrá una aceptación de la responsabilidad.

\subsection{Corpus y metodología}

El presente corpus está compuesto por un total de 1567 respuestas publicadas en la segunda quincena del mes de julio de 2017 en las cuentas oficiales de atención al cliente de dos empresas del IBEX 35, Iberia y Santander (Tabla 1). La muestra seleccionada representa un particular modo de expresión en un marco específico, el digital, acotado a un momento en el que gran parte de las quejas en las compañías se canalizan a través de las redes.

Santander (@Santander_resp) ha creado un perfil específico para atender las quejas y dudas de sus clientes, a diferencia de Iberia (@Iberia), que centraliza la información sobre la compañías y resolución de problemas en una misma cuenta. Así se entiende que Iberia esté a la cabeza con 529.000 seguidores, seguida por el Banco Santander (21500). Las dos empresas han sido seleccionadas por su comportamiento en redes sociales (Epsilon, 2016) dentro del Ibex 35. El diseño de la muestra nos permitía comparar el comportamiento en redes sociales de dos grupos distintivos (banca frente a compañías aérea) y al mismo tiempo se ofrecía una ocasión para estudiar de qué modo se comportaban dos modos distintos de canalizar la atención al cliente en Twitter, bien a través de perfiles específicos (caso del Banco Santander) bien atendiendo a través de uno general (caso de Iberia).

Los tuits se obtuvieron a través del programa Twitonomy ${ }^{2}$, software de analítica de Twitter. Cada dos días y a la misma hora se sondearon los perfiles de estas cuentas para obtener nuevos tuits; se descargaron hasta 2000. No fue necesario descartar ningún retuit, pues no aparecían al tratarse de respuestas personalizadas a tuits, pero sí se eliminaron las repeticiones encontradas, de tal manera que el corpus del Banco Santander quedó reducido a 567 tuits (Tabla 1).

El marco temporal seleccionado en el caso de la compañías aérea respondía a un momento complicado, dado el aumento de viajeros que se experimenta en los meses estivales; de ahí que el número de disculpas registradas sea más alto que el del banco Santander, donde en principio estamos ante meses tranquilos. La media que se registra es de 67 tuits por día en Iberia frente a los 38 tuits que encontramos en el caso del Banco Santander (Tabla 1).

La muestra presenta inevitables limitaciones: por un lado, hubiera sido plausible una muestra más equilibrada con dos subcorpus de casi igual número de palabras a la hora del análisis lingüístico; sin embargo, consideramos que esta diferencia arroja luces importantes

$2<$ https://www.twitonomy.com/> 
en cuanto a cómo es el comportamiento y uso de cada una de estas cuentas. Por otro, hubiéramos querido acceder a los diálogos completos que se producen entre cliente y empresa de modo automático, pero Twitonomy no nos permitía una descarga directa donde se recogiera la voz de ambas partes de modo continuo.

\begin{tabular}{lccc}
\hline & Tuits & Palabras & Tuits por día \\
\hline Iberia (@Iberia) & 1000 & 15222 & 67 \\
Santander(@Santander_resp) & 567 & 8443 & 38 \\
\hline Total & 1567 & 23655 & \\
\hline
\end{tabular}

Tabla 1. Corpus analizado.

Los tuits descargados se trataban de tuits dirigidos a personas concretas; como se indicó, estos tuits empiezan con el avatar del seguidor de twitter (@username) y pueden ser visto por los seguidores de la compañías dentro de la pestaña Tweets y respuestas, diseñada precisamente para dar visibilidad a cómo se atiende al cliente. Por cuestiones de confindencialidad se han eliminado los avatares particulares y sustituido por la palabra username. Asimismo se recogen los tuits tal y como aparecen publicados sin eliminar las faltas que pudieran exisitir (3).

(3) @Iberia He reclamado los puntos y me decis que el pasajero no se encuentra asociado a este vuelo (Cod. 30) 2/2

Se tomó como unidad de análisis el tuit, aunque con un pretest inicial aplicado a 100 tuits de cada uno de los corpus, se vio que un único tuit no equivalía siempre a una única disculpa. En ocasiones un tuit no ofrecía el espacio suficiente para una disculpa y este hecho explicaba que la intervención de la compañía se extendiera hasta a dos, tres o cuatro tuits (Tabla 2). Cuando así se producía, el tuit aparecía un número final /2, /3, /4 (4) o en el caso del Banco Santander 1/3, 2/3. De este modo el número de disculpas analizadas eran en el caso de Iberia 783 frente al Banco Santander, 431, tal y como se preveía puesto que el contexto temporal afecta de manera distinta a estos dos sectores.

(4) @username res Hola Leo, ¿has verificado en el formulario de objetos perdidos? si la tripulación recupera un objeto perdido, lo entrega en el /1 @username res aeropuerto para que suban los datos al formulario https://t.co/KhyHQtkVBh. Normalmente, hasta pasadas unas 24-48 horas /2@username res no saldría reflejada la info en ese formulario. Consulta el formulario entonces, a ver si consta ahí. Si no, puedes contactar /3@username res directamente con el Centro de Objetos Perdidos por mail, oex@iberia.es, indicando los detalles de tu vuelo y asiento. Saludos $/ 4$

\begin{tabular}{lccccc}
\hline & Disculpa=1 tuit & Disculpa=2 tuits & Disculpa $=3$ tuits & Disculpa=4 tuits & Disculpas totales \\
\hline Iberia & 783 & 89 & 9 & 3 & 783 \\
\hline Santander & 320 & 90 & 17 & 4 & 431 \\
\hline
\end{tabular}

Tabla 2. Número de tuits por disculpa. 
Para el análisis de las expresiones de disculpa se utilizó la metodología basada en análisis de la lingüística de corpus (Mair y Hundt, 2000) y la pragmática de disculpa (Harris et alii, 2006). Con el programa Sketch-Engine y las herramientas de concordancia que ofrecía se cuantificaron, en primer lugar, los lexemas con más frecuencia (Kilgarriff et alii, 2014). Pero este era tan solo un punto de partida ( $\$ 3.1)$; el análisis cuantitativo nos permitía abordar el análisis de un corpus relativamente grande y arrojaba ideas clave que, después, exigían ser analizadas con un posterior análisis cualitativo (Bednarek, 2008; Jucker and Taavitsainen, 2008 ). Las palabras con más presencia en los tuits, son las denominadas palabras clave positivas, pero también se prestó atención a aquellas que por comparación a otros corpus de referencia, en este caso, a los corpus de disculpas, no aparecían, son las denominadas palabras clave negativas (Rayson, 2008).

A continuación se fija la atención en los componentes que conforman las disculpas corporativas analizadas según la taxonomía expuesta en el apartado anterior; se comienza por los llamados MIFI (§ 3.2.1), siguiendo la propuesta de Siebold (2008) y Harris et alii (2006), y, a continuación, el resto de estrategias encontradas.

\section{RESULTADOS Y DISCUSIÓN}

Para dar respuesta a las preguntas de investigación planteadas, se presentan los datos obtenidos organizados en dos subapartados: lista de palabras clave y componentes de la disculpa. Dentro de los componentes, en primer lugar se presentan los MIFI, son lo que podríamos denominar disculpas explícitas (\$3.2.1) y, a continuación, el resto de componentes que apoyan de un modo implícito la disculpa ( $\$ 3.2 .2$ a $§ 3.2 .7)$.

\subsection{Palabras clave}

En primer lugar, se obtiene la lista de palabras con más frecuencia (Tabla 3). Normalmente entre las palabras con más frecuencia se encuentra artículos, preposiciones, conjunciones y verbos auxiliares. Eliminadas estas palabras sin entidad semántica, se presta atención a lo que ocurre por debajo de esas 20 primeras posiciones; es entonces cuando aparecen las palabras interesantes, las que deben tomarse en consideración, como los sustantivos, adjetivos y verbos (Tabla 4).

Fijándonos en la frecuencia, es relevante que en las dos compañías entre las 3 entradas más frecuentes se hallen saludos y hola, dos términos asociados al hecho dialógico que se reproduce en Twitter ${ }^{3}$. Así mismo destacan la presencia de por favor y gracias, dos expresiones vinculadas por excelencia a la manifestación de la cortesía, tan presente en el mundo de la disculpa.

Una diferencia llamativa se produce en el hecho de que el nombre de la compañía solo aparece en el caso del Banco Santander (30 ocasione); Iberia, sin embargo, decide no mencionarse explícitamente porque evita una política de marca marcada, al tener en cuenta que está respondiendo a quejas de clientes.

3 En $§ 3.2 .6$ se analiza más detenidamente. 


\begin{tabular}{lccc}
\hline \multicolumn{2}{c}{ SANTANDER } & \multicolumn{2}{c}{ IBERIA } \\
\hline Palabras & frecuencia & palabras & frecuencia \\
\hline de & 365 & Hola & 565 \\
por & 205 & Saludos & 514 \\
que & 190 & de & 413 \\
la & 180 & por & 279 \\
a & 161 & que & 266 \\
tu & 144 & en & 263 \\
Hola & 138 & a & 262 \\
en & 116 & la & 240 \\
el & 110 & tu & 229 \\
para & 107 & el & 211 \\
NH & 99 & con & 166 \\
te & 95 & los & 141 \\
AA & 95 & te & 128 \\
MG & 91 & y & 126 \\
y & 90 & lo & 125 \\
compañeros & 78 & nos & 122 \\
días & 76 & DM & 120 \\
DM & 74 & no & 114 \\
Buenos & 72 & un & 109 \\
\hline
\end{tabular}

Tabla 3. Lista de palabras con más frecuencia.

\begin{tabular}{lccc}
\hline \multicolumn{2}{c}{ SANTANDER } & \multicolumn{2}{c}{ IBERIA } \\
\hline Palabras & Frecuencia & Palabras & Frecuencia \\
\hline Hola & 138 & Saludos & 578 \\
Buenas/os & 135 & Hola & 565 \\
saludo & 98 & vuelo & 91 \\
días & 80 & favor & 85 \\
compañeros & 78 & compañeros & 71 \\
Santander & 30 & disposición & 43 \\
tardes & 55 & lamentamos & 43 \\
ayudarte & 46 & web & 41 \\
favor & 45 & mensaje & 41 \\
mensaje & 34 & caso & 40 \\
privado & 34 & sentimos & 39 \\
tarjeta & 55 & vía & 38 \\
ayudarán & 55 & información & 37 \\
servicio & 32 & reserva & 37 \\
Gracias & 29 & respondido & 36 \\
realizar & 28 & Gracias & 36 \\
cuenta & 24 & privado & 34 \\
banco & 20 & desconocemos & 33 \\
oficina & 20 & aeropuerto & 32 \\
\hline
\end{tabular}

Tabla 4. Lista de palabras con entidad semántica con más frecuencia. 


\subsection{Componentes de la disculpa}

\subsubsection{MIFI}

En segundo lugar y de acuerdo con la pragmática de la disculpa, se fija la atención en los lexemas convencionalmente reconocidos como MIFI tanto verbos performativos como partículas intensificadores, que expresan la disculpa abiertamente: perdón, siento/ sentimos, lamento/lamentamos y pido/pedimos disculpas. La frecuencia es resumida en la Tabla 5.

\begin{tabular}{lcc}
\hline & Iberia & Santander \\
\hline lamentamos & 58 & 0 \\
sentimos & 39 & 26 \\
perdón & 0 & 0 \\
perdona & 3 & 0 \\
disculpas & 9 & 13 \\
disculpa (vb) & 5 & 0 \\
Total & 104 & 39 \\
\hline
\end{tabular}

Tabla 5. MIFI.

Tal y como atestigua la Tabla 5, Iberia es la compañía que más frecuentemente utiliza uno de los lexemas (104 veces) frente a 67 veces en el Banco Santander (5) y (6). El primer dato, por tanto, que llama la atención es la baja frecuencia de fórmulas explícitas en el sector bancario, en contra de la H1, que suponía un elevado número de MIFIS en ambas cuentas.

\section{Banco Santander}

(5)@username Te pedimos disculpas. La carta intenta reflejar de lo manera más exacta la operación financiera que se va a realizar. (1/4)@ username En la ampliación por cada acción que tengas se te entrega un derecho, que es el modo de adquirir acciones nuevas de la(2/4)@username ampliación de capital a un precio de 4,85€ cada una y a cambio de 10 derechos. Estos derechos se negocian en la bolsa, (3/4) PA (4/4)

Iberia

(6) @ username Hola Samu, esperamos tengas un gran vuelo :D cualquier duda a tus órdenes, Saludos@ username Hola Damm, lamentamos leer lo que comentas, personal de mostrador así como Ibconecta te irán indicando cómo se procederá, Saludos

Respecto a las preferencias léxicas de las disculpas explícitas, dentro de la compañía Iberia se puede destacar el uso del lexema lamentamos (58 veces), que en ningún caso aparece en el Banco Santander. Por su parte, el banco prefiere los verbos performativos sentimos (26) o la expresión pedimos disculpas (23), que aparece representada en Iberia hasta en 9 ocasiones junto al verbo disculpa ( 5 veces). También cabe mencionar la ausencia de la palabra perdón, sería un caso de palabra clave negativa. Tan solo en Iberia y con una 
frecuencia muy baja es posible hablar del verbo perdona (3 veces), probablemente porque en las disculpas corporativas no se asumen los procesos actitudinales que conllevan el perdón: remordimiento y/o arrepentimiento (Konstan, 2011).

Otro elemento significativo diferenciador es que las palabras con poder de MIFI, que en otros contextos pueden aparecer aisladamente, no se produce en ningún tuit, puesto que necesitan una explicación, que implícitamente conlleva el asumir la responsabilidad. De esta manera se confirma la H3: en todas las disculpas habrá una aceptación de la responsabilidad. Por otra parte, en todos los casos, estas expresiones son ampliadas con más detalles propios de la disculpa o los actos de lengua adicionales, como veremos a continuación.

Frente a las diferencias citadas, es reseñable que no se utilicen adverbios intensificadores. Tan solo hemos encontrado un ejemplo en Iberia (7). Tal y como se anunciaba en la hipótesis 2, no hay ninguna tendencia a enfatizar el grado de responsabilidad en los discursos públicos, puesto que de ese modo la imagen de la empresa se ve erosionada.

(7) @username Hola Joaquin, lamentamos mucho los inconvenientes. Nuestros compañeros de aeropuerto os contactarán directamente una vez se.../1@ username ...asigne un nuevo horario de salida para el vuelo. Saludos.

\subsubsection{Declaración del problema}

Como paso siguiente, se distinguió si los tuits reproducían el problema explícitamente (8) o evitaban repetir la ofensa a través de elipsis o referencias catafóricas (esto, lo) o expresiones generalizadoras (p.ej. las molestias) (10) a (12). Tal y como se puede deducir de la Tabla 6, la tendencia no es repetir el problema, por dos motivos fundamentales: por una parte, es un modo de recordar las ofensas o daños provocados, una política que afecta negativamente a la imagen de la empresa puesto que recuerda los errores de sus productos o servicios; por otro, la brevedad del tuit obliga a seleccionar muy bien los datos aportados con un doble objetivos: ayudar a solucionar el problema y, sobre todo, mejorar la imagen de marca, a pesar de que como indica Deutschmann (2003:57) utilizar los deícticos provocan que la compañía se distancie de la persona afectada, si bien este distanciamiento se suple con otros recursos $(\S 3.2 .6)$.

(8)@username Hola Gabriela, en general, los mostradores se abren de 2 a 3 horas antes del vuelo, exceptuando Barajas dónde es /1@username posible facturar desde 24 horas antes de tu vuelo. Lamentamos si en este vuelo la espera en mostradores ha sido $/ 2 \ldots$

(9)@username Hola. Sentimos el trato recibido por parte de la oficina, desde el Banco Santander trabajamos para que todos nuestros (1/2)@pererierapaul clientes queden totalmente satisfechos con nuestro servicio. ¿Podemos ayudarte desde aquí a resolver tus dudas? ${ }^{\wedge} \mathrm{MG}(2 / 2)$

(10)@username Buenos días Eduardo. Para poder conocer más detalles de lo que nos comentas, ¿puedes pasarnos una captura del error que aparece?^ $\mathrm{MG}$

(11)@username Hola,sentimos loocurrido.

(12)@username Buenos días Carlos. ¿A través de que vía se dio de alta la incidencia que comentas? ${ }^{\wedge} \mathrm{MG}$ 


\begin{tabular}{|c|c|c|}
\hline & Iberia & Santander \\
\hline \multirow[t]{6}{*}{ Ofensa repetida } & equipajes (11) & malestar por la espera (13) \\
\hline & maleta (12) & trato recibido por parte de la oficina (3) \\
\hline & retraso $(7)$ & \\
\hline & cancelación (7) & \\
\hline & demora (6) & \\
\hline & vuelo de tus padres (1) & \\
\hline \multirow[t]{9}{*}{ Ofensa evitada } & lo $(23)$ & lo ocurrido (2) \\
\hline & & lo que comentas (1) \\
\hline & & lo que trasladas (2) \\
\hline & molestias (6) & malestar (8) \\
\hline & inconvenientes (19) & molestias (7) \\
\hline & incidencia (2) & inconvenientes (11) \\
\hline & lo que comentas (18) & caso (3) \\
\hline & escribes (5) & situación (7) \\
\hline & tu opinión (20) & incidencia (1) \\
\hline
\end{tabular}

Tabla 6. Mecanismos ante la ofensa.

\subsubsection{Presentación de explicaciones}

Las explicaciones de cómo se produjo la infracción funciona como disculpas, bien vayan acompañadas de recursos explícitos, bien lo hagan directamente. El comportamiento con respecto a las explicaciones dadas en el perfil de las compañías es distinto. Mientras Iberia se ve en la obligación de manifestar el porqué de los daños sufridos, en el caso del banco Santander esta práctica es casi inexistente: solo 4 ocasiones (13).

(13)@username Sentimos los inconvenientes que nos trasladas En ocasiones, se producen momentos de saturación en nuestras oficinas. (1/2)

Iberia entiende que una explicación predispondrá al viajero para aceptar mejor la disculpa, de esta manera su responsabilidad directa se minimiza o, incluso, desaparece (14). Sin embargo, la política del banco es muy distinta y no se siente en la obligación de dar explicaciones, en parte porque muchos de los tuits no responden a quejas, sino a petición de ayuda o información, y en este caso no se entiende que haya ofensa (15); y en otras ocasiones, cuando la situación es compleja porque prefiere canalizarlo a través del mensaje privado, donde el cliente pierde probablemente capacidad de reacción (16).

(14) @username Hola Rafa, lo sentimos pero con tan poco tiempo no podemos solicitar la acción. En caso de que comprase /1 @rafo_gar su vuelo con su tarjeta Iberia Plus, tendrán sus datos y la felicitarán directamente. Muchas gracias. Saludos $/ 2$

(15)@username Buen Día, soy nueva en el banco, intentó realizar una transferencia a un nuevo beneficiario,y dice que no tengo los permisos 
En respuesta a@username

¿Puedes ser que únicamente tengas el Acceso consultivo? Por favor, verifícalo (1/2)

(16)@username porque quitáis cajeros automáticos y la mitad de ellos ni funcionan? Me he andado $5 \mathrm{KM}$ para ir a un cajero que funcione.

Buenos días, sentimos las molestias ocasionadas. ¿Seguimos hablando por mensaje privado? $\wedge \mathrm{AA}$

Las explicaciones que se aportan son de distintos tipos: cuestiones que van más allá del control de la compañía o causas técnicas (17) a (20), o prácticas de la empresa (21) que han sido pensadas normalmente para beneficio de los clientes, tales como seguir un protocolo, un horario concreto, unas comisiones preestablecidas.

(17) @username Hola Ana, lo sentimos pero como te comentamos tienes que hablar con la aerolínea que operó el viaje para que pueda ayudarte.

(18) @username Hola Rafa, lo sentimos pero con tan poco tiempo no podemos solicitar la acción. En caso de que comprase /1 @rafo_gar su vuelo con su tarjeta Iberia Plus, tendrán sus datos y la felicitarán directamente. Muchas gracias. Saludos $/ 2$

(19)@username Hola Marc, lo sentimos pero nosotros aquí no tenemos lo que nos pides. Saludos

(20)@username Hola, no tenemos reportada incidencia alguna en el formulario en línea. Para descartar que sea problema del explorador/1@nanibucchi mediante el cual accedes, te pedimos intentar desde otro dispositivo (que no sea móvil) y probar borrando la memoria caché

(21) @username Hola Mathieu, lo sentimos pero no es posible ya que si no utilizas la ida se aplicará la /1@username clausula "No Show" donde el vuelo de vuelta se cancela automáticamente. Muchas gracias. Saludos

También como explicación que justifica lo vivido por el cliente se manifiesta qué se está haciendo para que no se vuelva a repetir esta circunstancia (22).

(22) @username Hola Jordi. Lamentamos los inconvenientes que te haya podido ocasionar el retraso en la entrega de tu equipaje. No obstante/1@ username te informamos que estamos trabajando@aena y@iberia para mejorar los procesos y reducir los tiempos. Esperamos que/2@username puedas ver los resultados en breve. Saludos./3

En el otro extremo, nos encontramos en ocasiones donde Iberia y, en esta ocasión también, el Banco Santander no ofrece ninguna explicación y solo se expresa un deseo por parte de las compañía de que se resuelva con la mayor brevedad posible o se ofrece para posteriores servicios o se pone en la mejor de las disposiciones para ayudar, o se deriva a otros compañeros o departamentos (ej. (23) (25). Por tanto, aunque no se acepte la responsabilidad directamente, nunca la empresa queda en mal lugar ni tampoco el cliente queda abandonado en el olvido, sino que, por el contrario, le sigue mostrando su preocupación con el objetivo de que la imagen no se vea afectada. Desde el punto de vista lingüístico, estamos más bien ante lo que podríamos denominar construcciones casi fijas, propias del ámbito de la cortesía, que quedan recogidas en la Tabla 7. 


\begin{tabular}{lcc}
\hline & Iberia & Santander \\
\hline Podemos ayudarte & 48 & 150 \\
Quedamos/nos ponemos/estamos a tu disposición & 43 & 19 \\
Seguimos/quedamos/nos tienes disponibles & 0 & 18 \\
Resolver con la mayor brevedad & 6 & 3 \\
Contactar con compañeros u otros departamentos & 71 & 134 \\
\hline
\end{tabular}

Tabla 7. Construcciones fijas.

(23) @username Hola Jean Carlos, desconocemos qué pudo pasar. ¿Hay algo en lo que podamos ayudarte? Saludos

(24) @username Hola Gonzalo, lamentamos los inconvenientes. Si podemos ayudar en algo quedamos a disposición. Saludos.

Santander

(25)@username Sentimos los inconvenientes. Nosotros quedamos disponibles para cualquier otra cuestión que quieras hacernos llegar.

\subsubsection{Ofrecimiento de recompensa}

Un procedimiento habitual para restaurar la imagen es el hecho de ofrecer recompensa. Esta práctia implica el reconocimiento del daño cometido y, al mismo tiempo, refleja las obligaciones legales de la compañía hacia sus clientes. Solo hemos constatado esta práctica en el caso de Iberia con una frecuencia de hasta 5 ocasiones, en todas ellas se ofrece un reembolso por el trastorno sufrido. En el caso de las compañías bancarias, cabe esperar que esta posible reparación se haga a través de mensaje privado, dado que los temas que se trata son mucho más delicados. Cuando se recoge esta situación, no aparece una declaración explícita del error cometido, sino que ya el propio reembolso es una prueba de la aceptación de la queja.

(26) @username ropa, artículos de higiene, te solicitamos guardes todas las facturas de modo que puedas enviárselas a los compañeros para 3/ @ratxelly que puedan darte el reembolso por esos gastos. Saludos.

(27)@username Hola William, la respuesta no será inmediata, ¿has enviado la documentación necesaria para solicitar/1@wlockward reembolso por tu expediente? Entendemos que es demora de equipaje ¿cierto? ¿Nos confirmas por MD? Saludos/2

En ocasiones el ofrecimiento de reparación llega a través de otros departamentos o compañeros, adonde la persona afectada no ha llegado bien por no saberlo, bien por no tener acceso. En este proceso podrían estar involucrados Atención al Cliente y otros líneas de Atención. Hasta en 134 ocasiones, en el Banco Santander se ofrece pedir ayuda a los compañeros, o bien acudir a la oficina (41 ocasiones). En el caso de Iberia (71), especifica qué compañeros le atenderán con el objetivo de calmar su preocupación: compañeros de aeropuerto, compañeros de equipaje, compañeros de atención al cliente.

(28)@username Hola María, ¿has hablado con nuestros compañeros de equipajes para que te puedan dar la última información sobre tu maleta? Saludos 
(29)@username Hola. Sentimos las molestias. Nuestros compañeros de @SantanderMx te ayudarán. Saludos. ^PA

\subsubsection{Promesa de mejora}

La promesa de mejora es una estrategia escasamente representada en los corpus seleccionados (30) y (31). La tendencia a evitarlas podría estar justificada si pensamos que estas acciones correctivas parecen más bien retórica hueca, propias del ámbito de la cortesía; con ellas, no se soluciona el problema particular del cliente, sino que por el contrario se siente más desatendido y enfadado. Esta indefensión puede acabar en tuits incendarios que se viralicen y dañen la imagen de marca.

(30)@username Buenos días Pablo. La opinión de nuestros clientes nos ayuda a mejorar, ¿quieres facilitarnos (1/2)@username tu documento de identidad por privado para que demos de alta una sugerencia al respecto? ¡Muchas gracias! SANTANDER

(31)@username Hola Cecilia, muchas gracias por la info. Sentimos lo ocurrido y tomamos nota para mejorar. Saludos IBERIA

\subsubsection{Prolongación de las acciones}

La tendencia de las compañías dentro del discurso de disculpa es pedir explicaciones y nuevas acciones que exigen salirse de Twitter. Esta práctica viene evidenciada a través la alta presencia de imperativos, preguntas, o verbos como recomendación o consejo. La Tabla 8 sugiere un comportamiento similar en ambas compañías.

\begin{tabular}{lcc}
\hline & Iberia & Santander \\
\hline Imperativos & 54 & 7 \\
Preguntas & 235 & 183 \\
Recomendar & 8 & 3 \\
Sugerir & 8 & 0 \\
\hline
\end{tabular}

Tabla 8. Modos de cortesía

Entre los ejemplos que podemos encontrar, tropezamos con situaciones en las que se pide aclaraciones de lo sucedido para poder actuar. Las respuestas a estas preguntas servirán para aclarar la naturaleza de la queja o la raíz del problema y ayudar en la solución, tal y como puede verse en los ejemplos siguientes:

(32) @username Hola Matias, ¿lo estás gestionando desde nuestra web? ¿Qué error te marca? ¿Nos cuentas por MD? Saludos

(33)@@username Buenos días Sergio. Para poder contestarte, ¿ De qué importe son esas liquidaciones? (1/2)@username ¿Puedes mandarnos una captura con ello? ${ }^{\wedge} \mathrm{MG}(2 / 2)$

(34)@username ¿Has intentado solicitarlo desde nuestra App de Banca Móvil, dentro de la opción "Contratación”? (2/3) @jazdaspain Hola José Antonio, ¿cuál es el nombre comercial de la cuenta que quieres cambiar? (1/3) @username Localizarás esta opción en el desplegable superior derecho de tu Posición Global. ¿Ya tienes descarga nuestra App? ^AC (3/3) 
En un porcentaje alto (hasta 199 veces), en Iberia se pide que se continúe las acciones a través del canal privado. En muchas situaciones, porque puede aparecer información confidencial que no debe salir la luz pública, como número de reclamación, reserva, datos particulares. En el Santander se produce un hecho parecido, hasta en 104 ocasiones se recurre a la privacidad de los mensajes privados o DM.

(35)@username Hola ¿nos contactas en un mensaje privado para indicarte detalles? Saludos

(36) @username Hola Ariana, ¿nos indicas los detalles de reserva en un mensaje vía DM? Saludos

En estos casos las preguntas pueden ser consideradas como un modo para no perder la reputación y mostrar la preocupación por el cliente proponiéndole una posible solución, si continúa con los pasos propuestos. Las acciones requeridas al cliente son solicitadas de un modo cortés y delicado gracias al uso de condicionales si, verbos modales puedes, podrías $\mathrm{y}$ fórmulas como por favor. Véase a continuación la frecuencia en Tabla 9 y ejemplos (37) y (38).

\begin{tabular}{lcc}
\hline & Iberia & Santander \\
\hline Si gustas, & 6 & 0 \\
Si lo deseas, & 6 & 1 \\
Si quieres & 6 & 5 \\
Por favor & 85 & 45 \\
Puedes & 55 & 85 \\
Podrías & 29 & 2 \\
\hline
\end{tabular}

Tabla 9. Modos de cortesía.

(37) @username Hola Fiona, ¿podrías indicarnos si has verificado con tu banco el cobro? Si gustas, puedes enviarnos más detalles por MD. Saludo

(38)@username Hola Jesús, lamentamos lo que nos indicas. Si podemos ayudar en algo, déjanos saber por favor. Saludos.

En todos estos casos tanto las preguntas como los imperativos tienen una doble función: por un lado, asistir físicamente a los clientes que se siente vulnerados, pero, por otro, dejar en el mejor de los lugares a la compañía, para que el cliente siga confiando en ella y la siga recomendando.

\subsubsection{Fórmulas de saludo, despedida y agradecimientos}

La estructura de Twitter, sin ser una red social pensada para el diálogo como puede ser el whatsapp, permite que dentro de cada uno de los tuits nos encontremos en un porcentaje muy alto con fórmulas de saludo, agradecimiento y despedida propias del entorno hablado. Los porcentajes, sin embargo, sugieren que el Banco Santander mantiene un comportamiento distinto al de Iberia: su comportamiento es más frío y alejado como lo atestigua la frecuencia en todos los valores siempre ostensiblemente inferior (Tabla 10). Iberia, sin 
embargo, entiende que es fundamental el personalizar para que el cliente se sienta exclusivo y, en última instancia, mejor atendido; de ahí la importancia de repetir el nombre de la persona que presentó la queja.

(39)@username Hola Cecilia, muchas gracias por la info. Sentimos lo ocurrido y tomamos nota para mejorar. Saludos IBERIA

(40)@username Hola Carlos. Sentimos la incidencia del cajero. ¿Quieres que continuemos la conversación por DM y nos cuentas con detalle? ${ }^{\wedge} \mathrm{MG}$

(41)@username ¡Hola! Nuestros compañeros de @santanderchile te ayudarán. Saludos.

Cuando la comunicación se realiza a través de dispositivos electrónicos siempre parece existir el temor a que no se sea atendido personalmente, sino por sistemas informáticos, que generan respuestas automáticas y generalizadas (Spencer-Oatey, 2008) y exentas de sinceridad (Brazeal, 2008: 148). Una respuesta, como la que intenta Iberia y también el Santander, aunque en menos ocasiones, siempre se percibe como auténtica y hace sentirse al cliente como el centro del proceso de la queja (Heritage y Clayman, 2011: 179). Incluso la fórmula de saludo repetida por Iberia hola resulta más cercana y espontánea que el saludo buenos dias, buenos tardes, más propio de un lenguaje más burocrático y formal, que prefiere el Banco Santander.

(42)@username Buenas tardes, para poder ayudarte, ¿nos puedes confirmar si eres cliente de Banco Santander en España? ^AA @ romasantablanco y nuestro departamento especializado estudiará tu caso. Un saludo.

(43)@username Hola Alejandro, para ver como ayudarte, ¿nos envías DM con los detalles de la reclamación? Saludos

(44) @username Buenos días, sentimos tu malestar. ¿Podemos ayudarte desde aquí? ¿Seguimos hablando por $\mathrm{DM}{ }^{\wedge} \mathrm{AA}$

Respecto al cierre, es digno de mención el uso de la firma, pero solo en el caso del banco Santander: cada tuit acaba con las iniciales del siguiente modo ${ }^{\wedge} A A$. Esta compañía entiende que este es un modo de generar confianza entre cliente y compañía al poner nombre y apellidos a la persona que está en el otro lado de la empresa; la firma permite seguir el itinerario de la comunicación con la seguridad de que la persona que inició la conversación la sigue, se hace responsable y, finalmente, la resolverá. Podemos decir que en este caso el tuit se presenta como un género más próximo al correo electrónico o a la carta.

\begin{tabular}{lll}
\hline & Iberia & Santander \\
\hline Hola & 566 & 138 \\
Buenos días & 4 & 72 \\
Saludos & 497 & 98 \\
Muchas gracias & 97 & 35 \\
Nombre propio & 572 & 63 \\
Firma & 0 & 422 \\
\hline
\end{tabular}

Tabla 10. Fórmulas de saludo, despedida y agradecimiento. 


\section{CONCLUSIONES}

Como ha podido comprobarse a partir de los resultados obtenidos el estudio confirma la validez de las cuentas corporativas de Twitter como corpus relevante para el estudio de la expresión de las disculpas en el servicio de atención al cliente de las empresas.

Desde el punto de vista de los elementos que componen la disculpa, cabe destacar un comportamiento similar en cuanto a la estructura del tuit se refiere; en líneas generales podemos decir que presentan saludo, cuerpo (frecuentemente con expresiones de agradecimiento) y despedida. Si bien en el caso de Iberia el trato con el cliente resulta más cercano e individualizado que el del Banco Santander: Iberia opta por la personalización con un uso elevado de nombres propios y el saludo informal hola, mientras el Banco Santander prefiere un lenguaje más burocratizado y alejado con el saludo buenos días, buenas tardes y con una firma final, que recuerda más a la práctica de las cartas o correos

Las disculpas en Twitter pertenecen al ámbito público y como consecuencia está altamente mediatizada, lo que explica que en ningún momento se niegue directamente la ofensa presentada. De esta manera la disculpa adquiere formas políticamente correctas en ambas compañías, como la referencia al problema a través de expresiones generales (molestias, incidencias, inconvenientes) o catafóricos (lo, esto), evitando en la mayoría de los casos repetir el problema.

La presencia de MIFI es mayor en el caso de Iberia, puesto que esta compañía atiende más quejas que el caso del Banco Santander; por dos razones: por una parte, los meses de verano son especialmente complejos para las compañías aéreas; por otra, el Twitter del Banco Santander atiende no solo quejas, sino también peticiones de información y ayuda en un porcentaje mayor a lo que ocurre en el caso de Iberia.

Respecto a las explicaciones como mecanismos indirectos de disculpas, de nuevo Iberia muestra una política mucho más preocupada por el viajero y entiende la necesidad de aportarlas, ya que estas la eximen de asumir la total responsabilidad. Por su parte, el Banco Santander, se muestra mucho más indiferente con el cliente y en raras ocasiones le ofrece una explicación.

Las disculpas se explican como estrategias de negociación y persuasión, y este hecho explica que muchas de las disculpas necesiten dos, tres o cuatro tuits, o que por motivos de confidencialidad o simplemente de necesidad de más explicación sea necesario salirse de este canal.

En suma, la disculpa se presenta como un acto de habla fundamental en cualquier ámbito, pero en el marco empresarial y dentro la comunicación digital corporativa, su necesidad se hace especialmente notable. Por ello, es necesario incluir corpus, clasificados y contextualizados, que sirvan al profesor EFE para abordar la disculpa como un tema clave en las relaciones empresariales, tanto en el entorno online como offline.

\section{Referencias bibliográficas}

Bednarek, M. (2008). "Semantic preference and semantic prosody re-examined", Corpus Linguistics and Linguistic Theory, 4(2), pp. 119-139.

Benoit, W. L. (2014). Accounts, excuses, and apologies: Image repair theory and research. Albany, NY: SUNY Press. 
Blum-Kulka, S., House, J., \& Kasper, G. (1989). Cross-cultural pragmatics: Requests and apologies (Vol. 31). Nueva York: Ablex Pub.

Brazeal, L. M. (2008). "The image repair strategies of Terrell Owens", Public Relations Review, 34(2), pp. 145-150.

Brown, P., \& Levinson, S. C. (1987). Politeness: Some universals in language usage (Vol. 4). Cambridge: Cambridge university press.

Coombs, W. T. (2007). "Protecting organization reputations during a crisis: The development and application of situational crisis communication theory", Corporate Reputation Review, 10(3), pp. 163-176.

Cosh, C. (2009). A man and his guitar. National Post. The Financial Post. Canada. https://goo.gl/ NhxX2V (20-10-2017).

Couso, R. P. (2005). Servicio al cliente: La comunicación y la calidad del servicio en la atención al cliente. Vigo: Ideaspropias Editorial SL.

Creelman, V. (2014). Sheer outrage: a rhetorical analysis of Lululemon's blog backlash. En Darics, E. (dir.). Digital Business Discourse. Basingstoke: Palgrave MacMillan.

Culnan, M. J., McHugh, P. J., \& Zubillaga, J. I. (2010). "How large US companies can use Twitter and other social media to gain business value", MIS Quarterly Executive, 9(4), pp. 243-259.

Deutschmann, M. (2003). Apologizing in British English. Umea: Umea University Press.

Eelen, G. (2014). A critique of politeness theory (Vol. 1). Abingdom: Routledge.

Epsilon (2016). La presencia corporativa de las compañias del Ibex 35 en las redes sociales: https:// goo.gl/79sNtx (20-06-2017).

Flynn, K. (2016). Twitter update shows how fast companies respond to your complaints. Mashable, 15 septiembre 2016: https://goo.gl/WLiMvo (20-07-2017).

Gruber, M. C. (2011). Pseudo-apologies in the news. En Yuasa, E., Bagchi, T. and K. Beals (Eds.), Pragmatics and Autolexical Grammar. Amsterdam, Netherlands: Benjamins, 93-105.

Hargie, O., Stapleton, K., \& Tourish, D. (2010). "Interpretations of CEO public apologies for the banking crisis: Attributions of blame and avoidance of responsibility", Organization, 17(6), pp. 721-742.

Harris, S., Grainger, K., \& Mullany, L. (2006). “The pragmatics of political apologies”, Discourse \& Society, 17(6), pp. 715-737.

Heritage, J., \& Clayman, S. (2011). Talk in action: Interactions, identities, and institutions (Vol. 44). Hoboken (Nueva Jersey): John Wiley \& Sons.

Holmes, J. (1998). “Apologies in New Zealand English”. En J. Cheshire and P. Trudgill (eds). The Sociolinguistics Reader: Gender and Discourse, Vol. 2, pp. 201-39. Londres: Arnold.

Hudson, S., Roth, M. S., Madden, T. J., \& Hudson, R. (2015). "The effects of social media on emotions, brand relationship quality, and word of mouth: An empirical study of music festival attendees", Tourism Management, 47, pp. 68-76.

Hull, K., \& Lewis, N. P. (2014). "Why Twitter displace broadcast sports media: A model", International Journal of Sport Communication, 7(1), pp. 16-33.

Hutchby, I. (2001). "Technologies, texts and affordances”, Sociology, 35(2), pp. 441-456.

Jucker, A. H., \& Taavitsainen, I. (Eds.). (2008). Speech acts in the history of English (Vol. 176). Amsterdam ; Philadelphia: John Benjamins.

Kampf, Z. (2009). "Public (non-) apologies: The discourse of minimizing responsibility", Journal of Pragmatics, 41(11), pp. 2257-2270.

Kilgarriff, A., Baisa, V., Bušta, J., Jakubíček, M., Kovář, V., Michelfeit, J., ... \& Suchomel, V. (2014). "The Sketch Engine: ten years on", Lexicography, 1(1), pp. 7-36.

Konstan, D. (2011). "Before Forgiveness: classical antiquity, early christianity and Beyond". Unpublished paper (online). Marzo, 12: https://goo.gl/V8SBRM (1-08-2017).

Kim, H., Park, J., Cha, M., \& Jeong, J. (2015). "The effect of bad news and CEO apology of corporate on user responses in social media", PloS one, 10(5), e 0126358.

Mair, C., \& Hundt, M. (Eds.). (2000). Corpus linguistics and linguistic theory (No. 1999). Rodopi. 
Meier, A., (1998). “Apologies: what do we know?", International Journal of Applied Linguistics, 8 (2), pp. 215-231.

Mills, S. (2003). Gender and Politeness. Cambridge: Cambridge University Press, Cambridge.

Mok, J., \& Tokunaga, M. (2009). "A cross cultural apology episode of a diplomatic repair: A study into Japan's former Prime Minister Koizumi's official apology in April 2005”, Journal of Language and Politics, 8(1), pp. 72-96.

Ogiermann, E. (2009). On Apologising in Negative and Positive Politeness Cultures. Amsterdam: John Benjamins Publishing Company.

Ott, B. L. (2017). "The age of Twitter: Donald J. Trump and the politics of debasement", Critical Studies in Media Communication, 34(1), pp. 59-68.

Pace, K. M., Fediuk, T. A., \& Botero, I. C. (2010). "The acceptance of responsibility and expressions of regret in organizational apologies after a transgression", Corporate Communications: An International Journal, 15(4), pp. 410-427.

Page, R. (2014). "Saying 'sorry': Corporate apologies posted on Twitter”, Journal of Pragmatics, 62, pp. 30-45.

Page, R., Barton, D., Unger, J. W., \& Zappavigna, M. (2014). Researching language and social media: A student guide. Abingdon: Routledge.

Park, J., Kim, H., Cha, M., \& Jeong, J. (2011). "Ceo's apology in twitter: A case study of the fake beef labeling incident by e-mart”, Social Informatics, pp. 300-303.

Parr, B. (2009). "How to: Use Twitter for customer service". Mashable.com. (11-05-2017).

Rayson, P. (2008). "From key words to key semantic domains", International Journal of Corpus Linguistics, 13(4), pp. 519-549.

Rundquist, S. (2007). “Apologies-form and function: 'I think it was your foot I was stepping on"”, Pragmatics And Beyond New Series, 155, pp. 293.

Salgado, E. F. (2012). La imagen social en la selección de las expresiones de disculpa. En EscamillaMorales, J., \& Henry-Vega, G. (eds). Miradas multidisciplinares a los fenómenos de cortesía y descortesía en el mundo hispánico. Barranquilla: Universidad del Atlantico, pp. 214-246.

Schultz, F., Utz, S., \& Göritz, A. (2011). "Is the medium the message? Perceptions of and reactions to crisis communication via twitter, blogs and traditional media", Public Relations Review, 37(1), pp. 20-27.

Schultz, H. (2011). El desafío Starbucks: cómo Starbucks luchó por su vida sin perder su alma. Madrid: Aguilar.

Searle, J. R. (1969). Speech acts: An essay in the philosophy of language (Vol. 626). Cambridge: Cambridge University Press.

Shariati, M., \& Chamani, F. (2010). "Apology strategies in Persian”, Journal of Pragmatics, 42(6), pp 1689-1699.

Siebold, K. (2008). Actos de habla y cortesía verbal en español y en alemán: estudio pragmalingüistico e intercultural (Vol. 42). Frankfurt am Main; New York: Peter Lang.

Spencer-Oatey, H. (2008). "Face, (im)politeness and rapport". En H. Spencer-Oatey (ed.), Culturally Speaking ( $2^{\mathrm{a}}$ ed.). Londres: Continuum, pp. 11-47.

Tanaka, N., Spencer-Oatey, H., \& Cray, E. (2008). Apologies in Japanese and English. Nueva York: Continuum International Publishing Group.

Thornborrow, J.; Montgomery, M. (2010). "Special issue on personalization in the broadcast news interview", Discourse and Communication, 4, (2), pp. 99-104.

Tzu Yu, L. (2015). Estudio pragmalingüístico español y chino de actos de habla expresivos: disculpas y agradecimientos: https://goo.gl/DMPfUj (06-08-2017).

Unceta Gómez, L. (2014). "Pedir perdón en latín. El acto de habla de la disculpa en las obras de Plauto y Terencio", Emerita: Revista de Lingüística y Filología Clásica, pp. 69-97.

Zappavigna, M. (2011). “Ambient affiliation: A linguistic perspective on Twitter”, New Media \& Society, 13(5), pp. 788-806. 